\title{
Effets De La Regulation Et Du Developpement Institutionnel Sur Le Secteur Bancaire De La Cemac
}

\author{
Jumbo Urie Eleazar \\ Faculté des Sciences Economiques et de Gestion, \\ Université de Bamenda, Cameroun \\ Ndzana Mekia Alain Bertrand \\ Doctorant en Economie, Faculté des Sciences Economiques et de Gestion, \\ Université de Dschang, Cameroun
}

doi: 10.19044/esj.2016.v12n1p300 URL:http://dx.doi.org/10.19044/esj.2016.v12n1p300

\begin{abstract}
The objective of this study is to assess the impact of the regulation (of requirements in equity capital) and of the institutional development on some performances of the "CEMAC' banking sector. The secondary data used come from the publications of the World Bank (World Bank Development Indicators) and from the services of 'BEAC' and 'COBAC', The method of estimation is that of the Generalized Moment Method (GMM) in dynamic panel via the model of Arellano and Bond (1991). The study spans a period of 8 years (2005-2012) and takes into account the six countries of the CEMAC subregion. The results of the study are presented as follows: (1) the level of the prescribed equity capital does not affect neither the "banking development" nor the "capacity of mobilization of the domestic saving" and just as the "economies financing capacity". (2) The level of legal and institutional development influences negatively and significantly the "banking development" and the "volume of domestic saving”. (3) Also, the level of legal and institutional development influences positively and significantly the "capacity of the banking sector to finance the economies". These results lead therefore to some implications of economic policy such as: the public authorities should reinforce the financing capacity of the "CEMAC" economies in setting up a real financial market. They should also develop and in a significant way political and institutional infrastructures in order to stimulate the confidence of savers and investors.
\end{abstract}

Keywords: Financial regulation; Legal and institutional development; Financial development; Dynamic panel 


\section{Resume}

L'objet de ce travail est d'évaluer l'impact de la régulation (des exigences en capitaux propres) et du développement institutionnel sur certaines performances du secteur bancaire CEMAC. Les données sont de source secondaire et proviennent de l'ensemble des publications de la Banque Mondiale (World Bank Development Indicators), des services de la BEAC et COBAC. La Méthode d'évaluation est celle des Moments Généralisés (GMM) en panel dynamique via le modèle d'Arellano et Bond (1991). L'étude s'étant sur la période 2005-2012 et prend en compte tous les six pays de la sous-région CEMAC. Les résultats se présentent ainsi qu'il suit: (1) le niveau des fonds propres réglementaires n'affectent ni le " développement bancaire », ni la "capacité de mobilisation de l'épargne intérieure » et non moins la "capacité de financement des économies ». (2) le niveau du développement institutionnel et légal influence négativement et significativement le " développement bancaire » et le " volume de l'épargne intérieure ». (3) Aussi, le niveau du développement institutionnel et légal influence positivement et significativement la « capacité du secteur bancaire à financer les économies ». Ces résultats donnent donc lieu à quelques implications de politique économique à savoir que : les autorités publiques doivent renforcer la capacité de financement des économies de la CEMAC par la mise sur pied d'un véritable marché financier. Elles se doivent aussi de développer impérativement et de manière considérable les infrastructures institutionnelles et politiques afin de stimuler la confiance des épargnants et des investisseurs.

Mots-clés : Régulation financière ; Développement institutionnel et légal ; Développement financier ; Panel dynamique

\section{Introduction}

Un système financier, développé, saint et bien diversifié est sans doute un facteur important de croissance pour les économies (Nord, 2011). Les entreprises disposent dans ce cas de grandes possibilités de lever les fonds dont elles ont besoin pour financer leurs activités. Les particuliers peuvent facilement épargner, ce qui permet d'accroitre la liquidité de l'économie.

La littérature oppose cependant l'approche keynésienne à celle dite classique en matière de développement des systèmes financiers et économiques. Dans le passé, et plus exactement après la grande dépression de 1929, bon nombre de pays se sont inclinés en faveur du modèle keynésien (l’interventionnisme) au détriment du modèle classique (le libéralisme). Ceci dans le but de stimuler le développement des systèmes financiers et économiques. Seulement, l'on peut noter que cette politique d'inspiration 
keynésienne s’est parfois pratiquée de façon très rigoureuse. Par exemple, elle s’est traduite au milieu des années 1980 par une réglementation stricte et généralisée, allant du plafonnement des taux d’intérêts, des restrictions à la création des succursales, des normes de fonds propres à des dispositifs d’assurance de dépôts. Au delà de cette dureté, les abus de certains gouvernements ont donné raison aux défenseurs du libéralisme à l'exemple de Mckinnon et Shaw (1973) qui ont vivement critiqué cette politique keynésienne. Seulement, la libéralisation financière elle aussi, est par essence porteuse d'instabilité financière avec des crises financières aux conséquences néfastes pour les économies et le bien-être des populations (Grosmann et Stiglitz, 1985 ; Minsky, 1982 ; Agglietta, 1991).

Comment remédier donc à la vulnérabilité du système financier ? En consultant la littérature financière, l'instrument le plus efficace de l'heure semble la prévention des risques systémiques par la régulation des capitaux propres des Banques définie par le comité Bâle dans les années 80 et 90. L'idée étant qu'un certain montant des capitaux propres évite la faillite bancaire et maintient la confiance dans le système. Mais, l'un des problèmes reste la détermination du niveau des fonds propres capable de réduire cette vulnérabilité du système financier sans autant compromettre son rôle essentiel qu'est le financement des économies.

Nombreux sont des auteurs qui ont mis en évidence le bien fondé de la régulation bancaire en termes de limitation du risque moral induit par l'assurance des dépôts et d'externalités liés à la faillite. La régulation est souhaitable pour limiter la prise de risques par les banques et asseoir la stabilité financière (John et al., 1994 et Saunders, 1985 ; Boyd et al., 1998 ; Beji et Youssef, 2010). Toutefois, comme l'a si bien remarqué Van den Heuvel (2008), les exigences du capital réglementaire n’ont pas que d'avantages; et si tel était le cas, pourquoi ne pas élever les ratios prudentiels à $100 \%$ et exiger que les actifs bancaires soient financés par les fonds propres ? En réalité, de telles mesures n’ont jamais pu être observées, laissant admettre implicitement que si les bienfaits des ratios prudentiels sont indéniables, il existe une limite à leur prescription, car le capital bancaire peut être à l'origine des prises de risques et des coûts induits (claessens et Klingebiel, 2000 ; Blum, 2007).

En plus des effets de cette forme de régulation bancaire, d'autres facteurs à caractère politico-institutionnel peuvent avoir une influence non négligeable sur le secteur bancaire et méritent une attention plus particulière. D’une façon générale, les économistes se sont penchés à la recherche des déterminants politico- institutionnels du développement des systèmes financiers. Ces facteurs définissent le degré de protection des investisseurs, les mécanismes d'application des contrats privés, la capacité du système légal à protéger les droits des propriétaires, ainsi que le développement des 
institutions financières compétitives et concurrentielles ${ }^{111}$. Selon cette perspective, ceux qui détiennent le pouvoir façonnent les politiques en vigueur ainsi que les institutions légales mêmes financières parfois à des fins personnelles ou égoïstes. Cette approche ne met pas en cause l'importance des institutions légales mais précise seulement qu’il existe des raisons politiques derrière des différences de niveaux de développement financier.

Dans le contexte africain en général et la zone CEMAC en particulier, la plupart des pays ont opté pour les accords de Bâle (1988) et la libéralisation financière (1990) après avoir appliqué la « répression financière $^{112}$ » au milieu des années 80, dans le cadre des programmes d'ajustement structurel mis en place par les institutions de «Bretton Woods ». Aujourd'hui, l'on assiste à un mélange de politiques de développement. Mais l'observation de la base de données proposée par Kaufmann et al. (2010) et les études empiriques de Rafael CEZAR (2012) montrent que les autorités de la CEMAC ont porté très peu d'intérêts au développement institutionnel et financier. D’où la mauvaise qualité des institutions et le faible niveau du développement financier. Contrairement donc aux pays de la CEMAC, les pays de l'Asie du Sud Est notamment, la Corée du Sud, la Malaisie, la Chine, le Chili ont adopté une politique alternative appelée le «séquencing ${ }^{113}$ " et des mesures visant le développement institutionnel et légal. Ce qui peut expliquer dans certaines études, la différence du niveau de développement financier et économique entre ces pays du Sud-Est Asiatique et ceux de l'Afrique Centrale.

Aujourd'hui, malgré cette symbiose de politiques de développement, le système financier de la CEMAC (essentiellement bancaire), ne parvient toujours pas à bien assurer le financement des économies de la sous-région dans un contexte de surliquidité bancaire et de besoins élevés de financement des Etats, des entreprises et porteurs de projets (conférence de Libreville du 13 juin 2013). On peut donc comprendre avec l'exemple des pays asiatiques que c'est la gestion des politiques de développement qui pose problème en zone CEMAC. Et donc, dans le cadre de cette étude, voyons si la régulation bancaire sous l'angle des exigences en capitaux propres et le développement institutionnel sont des facteurs sous-jacents à la situation de déficit important et persistant de financement en zone CEMAC. D’où la question centrale

\footnotetext{
${ }^{111}$ Pour plus d'explication, lire Pound (1991), Roe (1994), Pagano et Volpin (2001), Rajan et Zingales (2003), Habert et al. (2003).

${ }^{112}$ Pour Mckinnon (1970) et Shaw (1973), la répression financière consiste à fixer des taux d'intérêt en dessous de leurs valeurs d'équilibre, à orienter administrativement les crédits, à fixer des réserves obligatoires à des niveaux très élevés, et à limiter la concurrence au sein du système bancaire.

${ }^{113}$ C'est une démarche par étapes qui consiste de passer du protectionnisme à la libéralisation financière de manière progressive et non brutale.
} 
suivante : quel est l'impact des exigences en capitaux propres et du développement institutionnel sur le développement bancaire, la capacité de financement des économies et sur la capacité de mobilisation de l'épargne en zone CEMAC?

Pour répondre à cette question, l’organisation de l'étude s’articule autour de quatre sections. La première section sera consacrée à la clarification de quelques concepts et relations théoriques entre concepts. La seconde section présentera l'état des lieux du système financier de la CEMAC depuis les reformes réglementaires et institutionnelles de 1990. La troisième section s'intéressera à l'analyse économétrique sur données de panel (modèle d'Arellano et Bond, 1991) et la quatrième section présentera les résultats.

\section{Cadre conceptuel et théorique}

Cette section s'intéresse à quelques clarifications conceptuelles concernant la régulation financière, le développement institutionnel et le développement financier. Elle met aussi en évidence les différents liens théoriques voire empiriques qui peuvent exister entre ces différents concepts.

\section{Régulation financière}

Il existe un certain flou sémantique en ce qui concerne la régulation et cela mérite un éclaircissement. Les notions de régulation, de réglementation et de supervision financière sont le plus souvent employées indistinctement les unes les autres alors même qu'elles correspondent à des modes de contrôle différents qui ne se recouvrent pas totalement.

La régulation financière étant la notion la plus large, elle englobe : d'une part, l'ensemble des règles et des dispositions légales imposées par le législateur pour influencer sur le comportement des banques et des marchés financiers de manière à préserver la stabilité du système ; - et d’autre part, la surveillance du respect de ces règles par les différentes autorités compétentes (Darlani Tartari, 2002). Cette définition montre que la réglementation et la supervision constituent deux composantes de la régulation. Et bien entendu, lorsque cette régulation porte essentiellement sur le système bancaire, on parle de « régulation bancaire » qui dans cette étude sera limitée uniquement à la régulation en matière des capitaux propres des banques.

\section{Développement financier}

Le concept de « développement financier » ne fait pas l'unanimité. Néanmoins, cette notion peut s’appréhender à partir des différents points de vue de certains auteurs et de quelques critères macroéconomiques. Pour répondre à la question de savoir qu'est ce qu'un système financier développé ? Turrunç (1999) pense qu'un système financier est dit développé 
si et seulement si, il assure le fonctionnement d'un système de paiement efficace, solide, évolutif qui mobilise l'épargne et améliore son affectation à l'investissement. Le «Department of International Development, 2004 » souligne qu'un système financier est dit développé si l'on a des indicateurs suivants: les nivaux d'efficience et de compétitivité améliorés, le taux croissant des services financiers disponibles, une augmentation des institutions financières qui œuvrent dans le secteur, un accroissement du montant des liquidités disponibles, une augmentation de l'allocation des crédits privés, une amélioration de la régulation et de la stabilité du secteur financier, un aspect important pour la réduction de la pauvreté et le fait qu'une grande partie de la population peut davantage avoir accès aux opérations et services financiers. Pour Mckibbin (2005), un système est dit financièrement développé s’il est à la fois à mesure d'assurer la mobilisation de l'épargne privée, l'allocation efficiente des ressources, le renforcement de la liquidité du système, la diversification des risques, la réduction des coûts de l'information et de transaction, le rassemblement des capitaux à travers l'épargne individuelle des ménages et des bénéfices non distribués des entreprises. Au final, le facteur commun de ces définitions pour un système financier développé est en relation avec les opérations de mobilisation de l’épargne et de financement des économies.

\section{Développement institutionnel et légal}

C’est vers la fin des années 1990 et le début des années 2000 que certaines études empiriques et organisations internationales ont reconnu la nécessité du développement institutionnel ou de la bonne gouvernance dans l'activité économique. Selon kaufmann et al., (2010) par exemple, le développement institutionnel et légal d'un pays peut s'appréhender à partir des six critères suivants : l'expression et la responsabilité, la stabilité politique, l'efficacité du gouvernement, la qualité du cadre réglementaire, l'autorité de la loi et le contrôle de la corruption. D'après Rafael CEZAR (2012), le développement institutionnel, juridique et réglementaire d'un pays s’appréhende par la qualité des règles de droit et de la réglementation. Quels liens existent-t-ils entre ces trois concepts?

\section{Liens entre la régulation financière, le développement institutionnel et le développement financier}

Au regard de ce qui précède, l’on peut constater qu'il existe des liens théoriques entre la régulation et le développement financier d'une part; le développement institutionnel et le développement financier d’autre part. En quoi ces relations peuvent-elles se justifier? 


\section{Développement institutionnel et développement financier.}

Pour bénéficier par exemple de la libéralisation financière, les systèmes financiers du monde doivent impérativement se doter d'un cadre institutionnel et légal développé. Les économies qui manquent de systèmes légaux garantissant les droits de propriété et/ou l'application des contrats entre protagonistes, ne sont pas motivées aux opérations de prêts ainsi qu'à l'établissement des opérations financières. Les études de Claessen et al (2000) ainsi que celles de Caprio et al. (2004) montrent à ce sujet que, plus les créditeurs bénéficient des protections légales et plus le niveau de l'intermédiation bancaire se trouve développé. Ainsi, les protections légales des investisseurs, le niveau de crédibilité et la transparence des lois d'un système financier sont capables d'influencer les comportements des agents. En voici quelques approches qui expliquent davantage ce lien :

\section{Capital social}

Le terme « capital social » relève initialement de la sociologie et se rapporte aux avantages dont bénéficient les individus à partir de leurs adhésions à des associations ou à des communautés (Bourdieu, 1985). Il relève aussi du niveau de leurs relations humaines et de leurs capacités à progresser au sein de la société. Pour cette raison, Coleman (1990) et Gambetta (1988) montrent dans leurs travaux que, le capital social est un des déterminants de la performance des institutions d'une société. En justification de cette thèse, Putnam (1993) a montré aussi que le capital social est un déterminant de la performance des gouvernements régionaux italiens. L'originalité de cette approche est que le capital social est perçu comme un des facteurs qui influencent l'environnement institutionnel et légal. Ainsi, le capital social apparait comme un des déterminants significatifs du développement ou de la détresse bancaire. Il pourrait même dans certains cas expliquer les différences dans le développement financier et les performances économiques des pays.

\section{Théories de la loi et de la finance}

Les théories de la loi et de la finance quant à elles s'intéressent au rôle joué par l'environnement institutionnel et légal dans l'explication de la différence de niveaux de développement financier entre les pays (Beck et Levine, 2004). Cette approche montre qu'il existe un lien positif entre le système légal protecteur et le marché des capitaux. Elle met aussi en relation les traditions de législation et les comportements d'investissement dans différents pays. 


\section{Structures politiques}

Rajan et Zingales, (1998) pensent que l'état des systèmes financiers et légaux peut-être la conséquence directe des forces politiques du pays. Dans le souci d’éclairer au mieux cette pensée, Beck et al., (2003) tiennent compte des différences de systèmes politiques dans leur étude de la relation entre le cadre légal, institutionnel et les finances. Ils trouvent que l'origine légale explique les différences de niveau de développement des marchés financiers, du secteur bancaire et de protection des droits de propriété privé entre pays.

\section{Dotations naturelles}

Acemoglu et al. (2001) se sont focalisés sur l'état de l'environnement sanitaire des colonisés et les facteurs géographiques pour tirer quelques conclusions. Ils concluent à cet effet que, la stratégie d'implantation des colonisateurs est fonction des dotations dont bénéficie le pays colonisé. Et cela a des conséquences tangibles sur les institutions y compris les institutions financières. Ce constat fait par ces auteurs dans le cadre du développement institutionnel reste valable aussi pour le développement financier. En effet, dans une économie d'extraction, les occupants ne veillent pas à la mise en place et au développement des marchés financiers libres et compétitifs, puisqu'ils risqueraient de fragiliser leurs positions acquises. Par contre, dans une colonisation de peuplement par exemple, les occupants essaient d'y construire les institutions qui protègent les droits des propriétaires et contribuent donc au développement institutionnel et légal.

\section{Régulation financière et développement financier}

Dans le domaine commercial, les différentes institutions financières (le Fond Monétaire International, la Banque Mondiale) ont largement prodigué les pratiques de la libéralisation financière. Toutefois, ces institutions n’ont pas suffisamment souligné la nécessité de procéder par étapes. C'est ce qu'on appelle le processus de « sequencing » c'est-à-dire la démarche qui consiste à passer d'une politique de protectionnisme à une politique de libéralisation financière de manière progressive et non pas brutale. L’alternative est donc qu'une amélioration de la régulation bancaire pourrait être préférable à une politique d'encouragement à la concurrence bancaire en ce sens qu'on peut protéger les systèmes bancaires des pays en développement des mésaventures de la finance mondialisée, par essence porteuse d'instabilité financière.

Dans les économies en développement caractérisées par une insuffisance des marchés de capitaux, la plupart des firmes domestiques recourent au financement bancaire pour leurs projets d'investissement. Les banques répondent à ces demandes en collectant des dépôts auprès des 
épargnants. Apparemment simple, mais ce mode de financement qui est en réalité le principal dans les pays en développement est aussi problématique. En effet, les banques peuvent se retrouver incapables d'honorer toutes les demandes de retraits de liquidité émanant de leurs clients. C'est le risque d'illiquidité qui correspond à ces situations où la banque ne dispose pas de liquidité pour faire face à ses engagements. Ce risque découle principalement de la fonction de transformation d'échéances de la banque, qui amène celleci à avoir les emplois dont le terme est supérieur à celui de ses ressources. Il devient donc opportun pour des raisons de développement des systèmes de paiement et de règlement, de protéger des dépôts des clients des différentes banques au moyen des normes prudentielles.

\section{Etat des lieux de l'environnement financier de la CEMAC après les reformes réglementaires et institutionnelles de 1990.}

La Communauté Economique et Monétaire d'Afrique Centrale (CEMAC) est un ensemble de six pays : le Cameroun, le Gabon, la Guinée Equatoriale, la République Centrafricaine, la République du Congo et le Tchad. Sous la direction des institutions internationales, ces pays ont procédé à plusieurs reformes monétaires, financières et institutionnelles pour atteindre les objectifs macro-économiques de stabilisation financière et de croissance économique. Ces réformes ont peut-être modifié l'environnement financier mais les résultats restes très mitigés pour atteindre les objectifs escomptés.

\section{Paysage financier de la CEMAC et critiques}

Il est question ici de mettre en évidence quelques réformes monétaires, financières et institutionnelles effectuées par les autorités publiques depuis 1990.

\section{Réformes générales}

Les réformes monétaires et financières mises en place par les autorités publiques depuis 1990 ont consisté à la mise en œuvre des mesures internes et externes de la libéralisation financière.

En ce qui concerne les mesures internes nous pouvons citer comme Mpabe Bodjongo (2012) : - la libre fixation des taux d'intérêt par les banques en fonction des forces du marché tout en respectant le taux plafond et le taux plancher liés respectivement aux taux débiteur et créditeur - la suppression de la taxe sur la distribution du crédit - la suppression des taux d'escompte privilégiés par la banque - la programmation monétaire - la dévaluation du Franc CFA en 1994 - la création d’un marché monétaire - la liquidation des banques sinistrées et la fusion des banques - la capitalisation des banques et la réhabilitation des banques jugées viables - le 
désengagement de l'Etat dans la gestion active des banques commerciales la fixation des critères de délivrance des licences et des agréments aux dirigeants des banques - la libéralisation des commissions sur les transactions bancaires.

Quant aux mesures externes nous avons : - la création de la Bourse des Valeurs Mobilières de l'Afrique Centrale (BVMAC) et de la Douala Stock Exchange (DES) - la décision de la BEAC de ne plus racheter les billets exportés hors de la zone d'émission - la parité fixe entre le Franc CFA et le Franc Français (FF) et la garantie du Trésor Français d'assurer la convertibilité du FCFA.

\section{Réformes bancaires}

Il s’agit des réformes réglementaires, monétaires et institutionnelles visant à assurer une bonne réglementation et une bonne supervision bancaire. Sur ce, la COBAC et la COSUMAF ont été créées en 1992 et 2001 respectivement pour assurer la surveillance ou le contrôle des activités bancaires et des marchés financiers.

\section{Mise en place du dispositif prudentiel de la COBAC}

C’est à la suite d'un séminaire organisé par la BEAC à Libreville du 26 au 05 Avril 1990, qu’une reforme des règles prudentielles a été entreprise. Dans un premier temps, il avait été envisagé d’aligner immédiatement sur les règles internationales, l'ensemble des instruments prudentiels en vigueur dans la zone. Il est toutefois apparu au cours de la concertation avec les associations professionnelles des établissements de crédit qui a eu lieu au courant de l'année 1990, la nécessité de procéder par étape pour la mise en place de cette réforme. Pour des raisons de simplification et en fonction des résultats, le projet a été examiné lors du séminaire d'octobre 1992 tenu à Yaoundé sur la restructuration et la supervision des systèmes bancaires dans la zone. En conséquence, la Commission Bancaire de l’Afrique Centrale (COBAC) s'est dotée le 29 Mars 1993, de deux séries d'instruments lui permettant d'apprécier le comportement de certains établissements de crédit au regard des garanties de solvabilité et de liquidité qu'ils doivent présenter à tout moment. La solvabilité peut s'entendre comme l'aptitude d'un établissement de crédit à faire face en toute circonstance à ses engagements au moyen de ses ressources propres. Cette norme impose aux établissements de crédit le respect d'un certain nombre de ratios à savoir : le ratio de couverture des risques par les fonds propres fixé à $8 \%$ depuis $2006^{114}$; le ratio de couverture des immobilisations fixé à 100\% pour empêcher les banques d'affecter les dépôts au financement de leurs immobilisations et

\footnotetext{
${ }^{114}$ En 1992, ce ratio de couverture de risque était fixé à 5\%.
} 
donc à financer celles-ci par les fonds propres ou ressources permanentes ; le ratio de division des risques fixé à $75 \%$ et ramené à $45 \%$ le $1^{\text {er }}$ Janvier 1996 contre $25 \%$ pour la norme standard ${ }^{115}$, ; la limitation des prises de participation au capital des entreprises par les banques fixée à 15\% des fonds propres nets et donc l'ensemble ne doit excéder $75 \%$ et ramenée à $45 \%$ le $1^{\mathrm{er}}$ Janvier 1996 ; la limitation des concours octroyés par les établissements de crédit aux associés, actionnaires, dirigeants, administrateurs et le personnel fixée à 15\% des fonds propres nets. Par ailleurs, ces engagements excédant $5 \%$ des fonds propres nets viennent en déduction du montant de ceux-ci. La notion de liquidité quant à elle s'entend comme la capacité d'un établissement de crédit à honorer ses engagements à vue ou à très court terme. Elle repose sur deux ratios seulement : le ratio de liquidité générale fixé à $100 \%$ qui oblige les banques commerciales à disposer de manière constante les ressources immédiatement disponibles et susceptibles de couvrir au minimum l'intégralité des dettes à échoir dans un mois ; le ratio de transformation à long terme qui oblige les établissements de crédit à financer 50\% au moins des emplois à plus de cinq ans par les ressources de même terme. Ces exigences visent autant à assurer la sécurité des déposants qu’à préserver la valeur de la monnaie, notamment en veillant sur la qualité des crédits distribués et en garantissant la bonne marche de la profession. Toutes ces mesures ont un caractère individuel et vont de l'injonction au retrait d'agrément en passant par d’éventuelles astreintes financières pour dépassement de délai.

\section{Mise en place des réformes monétaires}

Au lancement du marché monétaire en Juillet 1994, des mesures sur la fonction de liquidité et la gestion des dépôts spéciaux prévoyaient que la BEAC pouvait injecter la liquidité quand elle juge nécessaire. Toutefois, elle pouvait aussi la retirer en offrant aux banques la possibilité d’ouvrir dans ses livres des comptes rémunérés selon les conditions du marché (dépôts spéciaux). Ces mesures ont prévalues jusqu’à la décision du gouverneur n001/MM/96 du 12 Janvier 1996, réaménageant le fonctionnement du marché monétaire.

C’est ainsi qu’à été institué le mécanisme de l’appel d’offre négatif en remplacement du système des dépôts spéciaux. Ce mécanisme s’oppose à l'appel d'offre positif. Sur le plan pratique, et en application des décisions du gouverneur $n^{\circ} 001 / \mathrm{MM} / 96$ du 12 Janvier 1996 et $n^{\circ} 003 / \mathrm{DCMM} / 96$ du 21 Février 1996, les innovations suivantes ont été introduites : - certains établissements financiers ont été admis au financement direct de la banque centrale (SOCCA, SOCABAIL). Ces établissements et des organismes

\footnotetext{
${ }^{115}$ En 1992, le ratio de division de risque était fixé à 75\%.
} 
publics détenteurs de dépôts spéciaux ont été éligibles aux appels d'offre négatif sous certaines conditions : - la modification du calendrier des appels d'offre afin de réduire certaines charges de fonctionnement du marché monétaire et rapprocher les offres des banques le jour de passation des écritures comptables, notamment le Jeudi ; - une nouvelle définition des taux créditeur minimum et débiteur maximum. En effet, les taux créditeurs et débiteurs sont désormais librement fixés par les banques qui doivent néanmoins respecter les limites. Le taux créditeur minimum appliqué aux petits épargnants est actuellement fixé à 3,25\% hors taxes l'an. Le taux débiteur maximum appliqué aux établissements éligibles aux concours de la BEAC est passé de $15 \%$ hors taxes l'an à presque $0 \%$ depuis 2008. Depuis 2012, la définition d'un taux d'usure par la BEAC vient compléter le dispositif étant attendu qu'il convient pour un emprunteur de tenir compte du taux d'effectif global. Pour renforcer la conformité des établissements de crédit aux règles établies, certaines résolutions réglementaires et normes prudentielles modifiant les pondérations applicables aux engagements sur les Etats avaient été prises au 31 Décembre 2010 : une nouvelle pondération des créances et engagements sur les Etats CEMAC et UEMOA en fonction du respect des critères de convergence définis dans la zone Franc, avait été adoptée. Les créances et engagements sur les Etats autres que ceux indiqués seront pondérés sur décision de la commission bancaire en fonction des éléments d'appréciation disponibles. Et les titres inscrits au bilan comme titres de transaction sont exclus de la détermination des normes de couverture et de division des risques durant le délai d'inscription au bilan dans cette catégorie de titres; délai qui ne saurait excéder six mois conformément au règlement de la COBAC R-2003/03 relatif à la comptabilisation et au traitement prudentiel des opérations sur titres réalisées par les établissements de crédit.

\section{Critiques}

Contrairement aux pays Asiatiques, l'environnement institutionnel et légal de la CEMAC reste caractérisé par: une pratique accrue de la corruption; une instabilité politique suivie des coups d'état, les guerres civiles...; une inefficacité de l'action gouvernementale marquée par une incompétence de certains fonctionnaires, une mauvaise qualité du service public, une bureaucratie peu avancée, la non crédibilité des engagements des décideurs publics et la dépendance vis-à-vis des pressions politiques; un manque d'expression et de responsabilité des citoyens qui s'observe à travers l'absence de la liberté de presse ainsi que le non respect des libertés civiles et des droits politiques ; un cadre réglementaire de mauvaise qualité avec une supervision insuffisante des activités financières et le non respect de la réglementation ; un faible indice de l'autorité des lois qui vient du fait que 
les individus n’ont pas confiance aux lois qui régissent la société et ne les respectent même pas ; les droits de propriété des investisseurs sont violés.

Les statistiques relatives à la régulation en matière des fonds propres des banques ne justifient pas un climat favorable au système bancaire CEMAC. Ainsi, les statistiques (COBAC) ${ }^{116}$ ressortent qu’en fin Juin 2011, 21 banques seulement sur 44 analysées (moins de la moitié), disposaient des fonds propres nets suffisants pour honorer à l'ensemble des normes assises sur cet agrégat contre 27 sur 45 l’année précédente à la même date. La norme prudentielle respectée par le plus grand nombre des banques est celle relative à la représentation du capital minimum. La norme liée à la limitation des risques encourus sur un même bénéficiaire constitue celle à l'égard de laquelle est observé le plus grand nombre des banques en infraction. Ces statistiques semblent une fois de plus justifier une certaine vulnérabilité du système bancaire CEMAC.

\section{Analyse économétrique}

Cette section expose la méthode d'estimation (3-1), les modèles à estimer (3-2), les variables d’étude et les tests préliminaires (3-3)

\section{Méthode d'estimation}

Il s’agit de la Méthode des Moments Généralisés (GMM) en panel dynamique (modèle d'Arellano et Bond, 1991). Cette méthode repose sur les conditions d'orthogonalité entre les variables retardées et le terme d'erreur en différence première ou en niveau. Lorsque le modèle dynamique est exprimé en différence première, les instruments sont en niveau et vice versa. L'utilisation des variables retardées comme instruments diffère selon la nature des variables explicatives. Pour les variables exogènes, leurs valeurs courantes sont utilisées comme instruments. Pour les variables prédéterminées ou faiblement exogènes (variables qui peuvent être influencées par les valeurs passées de la variable dépendante, mais qui restent non corrélées aux réalisations futures du terme d’erreur), leurs valeurs retardées d'au moins une période peuvent être utilisées comme instruments. Pour les variables endogènes, leurs valeurs retardées de deux périodes et plus peuvent être des instruments valides. La validité des instruments utilisés peut être confirmée ou infirmée à partir des tests de Hansen et de Sargan associés aux résultats des estimations. A cet effet, il existe deux formes d'estimateurs GMM en panels dynamiques : le modèle d'Arellano et Blond (1991) propose

\footnotetext{
${ }^{116}$ Statistiques COBAC de la $16^{\mathrm{e}}$ assemblée du comité des superviseurs des banques de
} l’Afrique centrale et de l'ouest, tenue à Conakry du 13 au 15 décembre 2011. 
un estimateur GMM en différence première. Il consiste à prendre pour chaque période, la différence première de l'équation à estimer pour éliminer les effets spécifiques des individus, et à instrumenter par la suite les variables explicatives de l’équation en différence première par leurs valeurs en niveau retardées d'une période ou plus. Ce modèle est le suivant :

$$
\Delta Y_{i t}=\beta \Delta Y_{i t-1}+\varphi \Delta X_{i t}+\Delta \varepsilon_{i t}
$$

Par contre, le modèle de Blundel et Bond (1998) détermine un estimateur GMM en système qui combine les équations en différence première et les équations en niveau dans lesquelles les variables sont instrumentées par leurs différences premières ou en niveau. Ce qui conduit au modèle suivant :

$$
\left\{\begin{array}{c}
\Delta Y_{i t}=\beta \Delta Y_{i t-1}+\varphi \Delta X_{i t}+\Delta v_{t}+\Delta \varepsilon_{i t} \\
Y_{i t}=\beta Y_{i t-1}+\varphi X_{i t}+v_{t}+\varepsilon_{i t}
\end{array}\right.
$$

Le choix de cette méthode se justifie par les avantages suivants : un estimateur GMM permet d'analyser les comportements dynamiques des variables et l'hétérogénéité entre les individus; d'éliminer les effets spécifiques des individus et d’instrumenter les variables explicatives par leurs valeurs en niveau retardées d'une période ou plus; de résoudre les problèmes de biais de simultanéité, de causalité inverse et de variables omises. A l'inverse, les techniques économétriques standards comme les moindres carrés ordinaires (MCO) ne permettent pas d'obtenir des estimations sans biais d'un modèle ayant une variable retardée à droite de l’équation.

Toutefois, contrairement à un estimateur GMM en système, l'estimateur GMM en différence première peut donner des résultats biaisés dans le cas des échantillons finis et surtout si les instruments sont faibles.

\section{Modèles économétriques}

Nos modèles s'inspirent donc de celui d'Arellano et Bond (1991). Cette approche a été utilisée par plusieurs auteurs comme Mpabe Bodjongo (2012) et Fouda Ekobéna (2014) pour ne citer que les plus récents. Nos modèles s’inscrivent donc ainsi qu'il suit:

\section{Modèle (1)}

$\Delta D B_{i t}=\alpha_{1} \Delta D B_{i t-1}+\alpha_{2} \Delta D I_{i t}+\alpha_{3} \Delta R B_{i t}+\alpha_{4} \Delta P I B H_{i t}+$ $\alpha_{5} \Delta I N F_{i t}+\Delta \varepsilon_{i t}$

De manière spécifique, ce premier modèle met en relation la variable dépendante développement bancaire $\left(\boldsymbol{D} \boldsymbol{B}_{\boldsymbol{i t}}\right)$ avec les variables explicatives que sont : la régulation bancaire $\left(\boldsymbol{R} \boldsymbol{B}_{i t}\right)$ et le développement institutionnel $\left(\boldsymbol{D} \boldsymbol{I}_{\boldsymbol{i t}}\right)$ en présence des variables macro-économiques de contrôle telles que l'inflation $\left(\boldsymbol{I N} \boldsymbol{F}_{i t}\right)$ et le produit intérieur brut par habitant $\left(\boldsymbol{P I B H} \boldsymbol{H}_{\boldsymbol{i t}}\right) ; \boldsymbol{\varepsilon}_{\boldsymbol{i t}}$ est le terme d'erreurs et $\Delta$ est l'opérateur de 
différence. Les $\boldsymbol{\alpha}_{\boldsymbol{j}},(\mathrm{j}=1 \ldots \ldots \ldots \ldots . .5)$ sont des paramètres à estimer. L’indice i désigne le pays concerné et $\mathrm{t}$ indique le temps.

Modèle (2)

$\Delta C F_{i t}=\alpha_{1} \Delta C F_{i t-1}+\alpha_{2} \Delta D B_{i t}+\alpha_{3} \Delta D I_{i t}+\alpha_{4} \Delta R B_{i t}+\alpha_{5} \Delta P I B H_{i t}+$ $\alpha_{6} \Delta I N F_{i t}+\Delta \pi_{i t}$

Ce second modèle met en rapport la capacité du secteur bancaire à financer l'économie du pays i à l'instant t $\left(\boldsymbol{C} \boldsymbol{F}_{\boldsymbol{i}}\right)$ et les variables explicatives que sont: la régulation bancaire et le développement institutionnel

$\left(\boldsymbol{R} \boldsymbol{B}_{\boldsymbol{i t}}\right.$ et $\left.\boldsymbol{D} \boldsymbol{I}_{\boldsymbol{i} \boldsymbol{t}}\right)$, en présence des mêmes variables de contrôle plus celle du développement bancaire $\left(\boldsymbol{D} \boldsymbol{B}_{i t}\right)$.

Modèle (3)

$\Delta M E I_{i t}=\alpha_{1} \Delta M E I_{i t-1}+\alpha_{2} \Delta D B_{i t}+\alpha_{3} \Delta D I_{i t}+\alpha_{4} \Delta R B_{i t}+$ $\alpha_{5} \Delta P I B H_{i t}+\alpha_{6} \Delta I N F_{i t}+\Delta \rho_{i t}$

Ce dernier modèle met en relation, la capacité du secteur bancaire à mobiliser l'épargne intérieure du pays i en temps t $\left(\boldsymbol{M} \boldsymbol{E} \boldsymbol{I}_{\boldsymbol{i t}}\right)$ et les variables explicatives $\boldsymbol{R B}_{\boldsymbol{i t}}$ et $\boldsymbol{D I}_{\boldsymbol{i t}}$ en présence des mêmes variables macroéconomiques de contrôle. $\boldsymbol{\pi}_{\boldsymbol{i}} \boldsymbol{e t} \boldsymbol{\rho}_{\boldsymbol{i t}}$ sont les termes d'erreurs dans les modèles (2) et (3) ; les $\boldsymbol{\alpha}_{\boldsymbol{j}},(\mathrm{j}=1 \ldots \ldots \ldots 6)$ sont les paramètres à estimer.

\section{Description des variables et tests préliminaires}

Notre échantillon est composé de six pays de la CEMAC à savoir : le Cameroun, le Gabon, la Guinée Equatoriale, la République Centrafricaine, la République du Congo, et le Tchad. Notre période d’étude va de 2005 à 2012. Le choix de cet intervalle de temps est lié à la disponibilité des données. En effet, les données sur le développement institutionnel et légal des pays de la CEMAC ne sont exposées que jusqu'en 2012. Ce qui nous empêche d’étendre notre étude à une période beaucoup plus récente.

\section{Présentation des données et variables}

Les données utilisées sont relatives au développement institutionnel, à la régulation en matière des capitaux propres, au développement bancaire, à la capacité de financement du secteur bancaire, à la capacité de mobilisation de l'épargne, au développement économique et à l'inflation. Elles sont de source secondaire et proviennent de l'ensemble des publications de la Banque Mondiale (World Bank Development Indicators), des services de la BEAC et COBAC. Ces données sont constituées en panel (cylindré) pour l'ensemble des six pays de la CEMAC. En ce qui concerne nos variables, certaines d'entre elles sont des indices composites et s'expriment comme des moyennes arithmétiques des variables de base : 


\section{Indice composite de développement bancaire (DB)}

Le choix du développement bancaire au lieu du développement financier s'explique par l'importance relative du secteur bancaire par rapport aux marchés des capitaux dans le fonctionnement de tout le secteur financier dans les pays étudiés. Il convient aussi d'insister sur l'intérêt particulier d'isoler la régulation en matière des capitaux propres du système bancaire. En effet, plusieurs indicateurs de développement bancaire ont été recensés dans la littérature économique. Mais, cette étude ne fait recours qu’à trois indicateurs retenus par Demétriades et Law (2005) sur le développement bancaire. Ces indicateurs sont : le crédit intérieur ou domestique fourni au

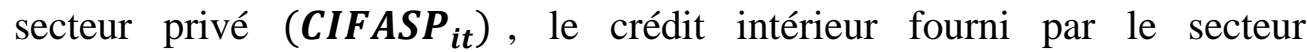
bancaire $\left(\boldsymbol{C I F P S} \boldsymbol{B}_{i t}\right)$ et les " passifs liquides » $\left(\boldsymbol{P} \boldsymbol{A S L I} \boldsymbol{Q}_{i t}\right)$. Ces indicateurs sont en pourcentage du PIB. Mais, dans cette analyse, faute des données concernant les "passifs liquides » pour l'ensemble des pays de la CEMAC, nous avons utilisé les données relatives à l'épargne intérieure qui est aussi un déterminant du développement bancaire comme l'ont révélé Turrunc (1999) et Mckibbin (2005). Ces données proviennent de l'ensemble des publications de la Banque Mondiale (World Bank Development Indicators) et des services BEAC. Cet indice synthétique capte globalement le développement bancaire en zone CEMAC et s'obtient comme une Moyenne Arithmétique des trois variables présentées ci-dessus :

$$
D B_{i t}=\frac{1}{3}\left(C I F P S B_{i t}+C I F A S P_{i t}+E P I_{i t}\right)
$$

Il est opportun de rappeler aussi que, le développement du système bancaire renvoie à une forte mobilisation de l'épargne domestique et à une allocation efficiente des ressources à l'économie par les établissements de crédit. A cet effet, les premières variables à savoir : «le crédit intérieur fourni par le secteur bancaire (CIFPSB) » et « le crédit intérieur fourni au secteur privé (CIFASP) » permettent de mesurer la capacité du secteur bancaire à financer les économies notée $\mathbf{C F}_{\mathbf{i t}}$. Par contre, la variable «épargne intérieure (Ep. I ) » permet de mesurer la capacité de mobilisation de l'épargne domestique notée $\mathbf{M E I}_{\mathbf{i t}}$. Ces deux indicateurs se présentent comme suit : $\boldsymbol{C F}_{i t}=(\boldsymbol{C I F P S B}+\boldsymbol{C I F A S P}) / \mathbf{2} ; \quad \boldsymbol{M E} \boldsymbol{I}_{i t}=E \boldsymbol{E p} . \boldsymbol{I}_{i t}$

\section{Indice composite du développement institutionnel et légal (DI)}

La prise en compte du développement institutionnel et légal dans cette analyse tient du fait que le développement des infrastructures institutionnelles, légales voire politiques influe très positivement sur l'approfondissement financier. Cet indice est construit à partir de six variables retenues de la base de données de Kaufman et al., (2010) et extraite de l'ensemble des publications de la Banque Mondiale (World Bank Development Indicators). Ces variables caractérisant l'environnement 
institutionnel et légal sont les suivantes: l'autorité de la loi (AL), la qualité de la réglementation (QR), la stabilité politique (SP), l'efficacité gouvernementale (EG), le contrôle de la corruption (CC), l'expression et la responsabilité (ER). L'indice synthétique de celles-ci s'inscrit comme une Moyenne Arithmétique :

$$
D I_{i t}=\frac{1}{6}\left(A L_{i t}+Q R_{i t}+S P_{i t}+E G_{i t}+C C_{i t}+E R\right)
$$

\section{Variable régulation bancaire (RB)}

La régulation bancaire repose ici sur la régulation en matière des capitaux propres et non sur la régulation financière d'une manière générale. Il est donc possible, d'étudier l'impact de cette variable sur le développement bancaire et non sur le développement financier. Ce choix se justifie par le fait qu'elle peut aussi être représentative du contrôle des capitaux propres exercé par les pays de la sous région. Ainsi, la norme prudentielle qui est prise en considération ici, limite l'exposition aux risques que peuvent prendre les banques domestiques et joue donc le rôle d'un contrôle sur les fonds propres des banques de la sous région. Cet indice représenté sous la forme logarithme du niveau des fonds propres réglementaires est défini par : $R B_{i t}=L n_{-} N F P R_{i t} \quad(\mathrm{e})$

\section{Variables macro-économiques de contrôle}

Nous avons ici le PIB par habitant qui capte le développement économique et l'inflation (INF) qui tient compte de la politique monétaire. Les données de ces variables proviennent des services BEAC. Le choix de ces variables tient du fait que : la variable PIB par habitant peut poser un problème de causalité inverse dans cette analyse. Toutefois, l'utilisateur de l'estimateur GMM nous permet de pallier ce handicap. La prise en compte de la variable inflation dans cette analyse tient du fait qu'elle est susceptible d'influencer les décisions économiques notamment en matière de placements et permet aussi d'intégrer les mesures de politique monétaire.

\section{Tests préliminaires}

Avant de procéder à l'estimation par la méthode d'Arellano et Bond (1991), nous avons fait recours à un ensemble de tests préliminaires sur les données notamment, les tests de stationnarité, les tests d'auto-corrélation des erreurs et le test de validité des instruments associés en panel dynamique.

\section{Tests de stationnarité}

Trois différents tests de racine unitaire courants ont été conduits pour vérifier la stationnarité des variables : le test de Levin-Lin-Chu (2002), le test Im-Pesaran-Shin (2003) et celui de Hadri (2000). Ces différents tests se 
distinguent par le niveau d’hétérogénéité introduit dans l’hypothèse alternative : moins restrictif pour le premier, le second relâche l'hypothèse d’homogénéité alors que le dernier se distingue par l'hypothèse nulle de stationnarité. Les procédures de ces tests de racines unitaires sont les suivantes : le test de Levin-Lin-Chu (LLC) a pour hypothèses $\boldsymbol{H}_{0}$ : les panels contiennent des racines unitaires contre $\boldsymbol{H}_{\boldsymbol{a}}$, les panels sont stationnaires; Décision : si la réalisation de la statistique corrigée de Levin-Lin-Chu est inférieure au seuil de la loi normale centrée réduite $(-1.64$ pour un test non symétrique à 5\% de risque de première espèce), l'hypothèse nulle de racines unitaires est rejetée pour l'ensemble des individus du panel. Pour le test d'Im-Perasan-Shin (IPS), nous avons l'hypothèse $\boldsymbol{H}_{0}$ : tous les panels contiennent des racines unitaires contre $\boldsymbol{H}_{\boldsymbol{a}}$ : quelques panels sont stationnaires. Décision : à 5\% de risque, si la réalisation de z-t-bar est inférieure au seuil de la loi normale centrée réduite (-1.64), l’hypothèse nulle de racines unitaires est rejetée. En ce qui concerne le test d'Hadri $(\mathrm{H})$ l'hypothèse $H_{0}$ : tous les panels sont stationnaires contre $H_{a}$ : quelques panels contiennent des racines unitaires. Décision : toujours à 5\% de significativité, si la p-value est inférieure à 0.05, l’hypothèse nulle de la stationnarité est rejetée.

\section{Tests d'autocorrélation des erreurs et de validité des instruments}

Deux tests sont associés à l'estimateur des GMM en panel dynamique et sont fournis avec les résultats de l'estimation : le test de suridentification de Sargan qui permet de valider les instruments utilisés dans les régressions en données de panel et le test d'Arellano et Bond qui permet de valider l'absence d'autocorrélation des erreurs. Le test de Sargan (1958)

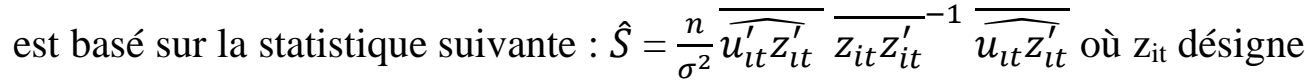
la matrice des instruments utilisés. La statistique du test suit la loi de ChiDeux à $(\mathrm{r}-\mathrm{k})$ degré de liberté, avec $\mathrm{r}$ le nombre d’instruments et $\mathrm{k}$ le nombre de paramètres à estimer. Ce test a pour $\underline{\text { Hypothèses }} \mathrm{H}_{0}: E\left(z_{i t}^{\prime}\left(y_{i t}-\right.\right.$ $\left.\left.x_{i t} \tilde{\beta}\right)\right)=0$, les instruments sont valides; $\mathrm{H}_{1}: E\left(z_{i t}^{\prime}\left(y_{i t}-x_{i t} \tilde{\beta}\right)\right) \neq 0$, les instruments ne sont pas valides. La décision est la suivante : si la P-value est inférieure à un certain seuil de significativité $\alpha$ égal à 1\%, 5\% ou $10 \%$ alors l'on rejette $\mathbf{H}_{\mathbf{0}}$. Dans le cas contraire, l'on ne rejette pas $\mathbf{H}_{\mathbf{0}}$. Le Test d'Arellano et Bond (1991) postule comme hypothèses: H0, absence de corrélation d'ordre 2 des résidus; $\mathrm{H}_{1}$, corrélation négative d'ordre 1 des résidus. Décision: si la $P$-value est inférieure à un certain seuil de significativité $\alpha$ égal à $1 \%, 5 \%$ ou $10 \%$ alors rejeter $\mathbf{H}_{\mathbf{0}}$. Dans le cas contraire, ne pas rejeter $\mathbf{H}_{\mathbf{0}}$. 


\section{Résultats}

\section{Résultats des tests préliminaires}

Malgré les limites que peuvent constituer le nombre réduit d’individus et de périodes à la validité des propriétés asymptotiques des statistiques des tests, les différents tests de racines unitaires utilisés affichent quelques différences selon le type. Mais les conclusions sont concordantes sur la présence d'une racine unitaire dans l'ensemble des variables hormis l'inflation. Nous avons écarté ces écueils dans l'estimation en utilisant les différences premières des variables de base. Ce qui par ailleurs a réduit le nombre d'observations de l'étude de quarante huit à trente. De plus, il ne serait pas très cohérent de chercher à saisir les relations de long terme et contenter du court terme. Les résultats des tests d'autocorrélation des erreurs et de validité des instruments utilisés dans les estimations sont fournis avec les résultats des estimations des différents modèles et confirment l'absence d'autocorrélation des erreurs et valident les instruments utilisés. Les tests de Wald fournis également avec les résultats confirment la bonne qualité des estimations en termes de significativité globale.

\section{Résultats des estimations des modèles et interprétation Résultats}

Tableau 1 : effets de la régulation en matière des capitaux propres et du développement institutionnel et légal sur le développement bancaire en zone CEMAC

MODELE 1

\begin{tabular}{|c|c|c|c|c|}
\hline \multirow{3}{*}{$\begin{array}{c}D_{-} D B \\
D_{-} D B_{\text {retardé }}\end{array}$} & coefficient & standard error & $Z_{c a l}$ & $p>\left|z_{\text {cal }}\right|$ \\
\hline & 0.2418228 & 0.0600255 & 4.03 & $0.000^{* * * * *}$ \\
\hline & -0.0845512 & 0.0278339 & -3.03 & $0.002^{* * *}$ \\
\hline$D_{\_} R B$ & 4.386362 & 2.934165 & 1.49 & 0.135 \\
\hline D_PIBH & 10.23715 & 5.309317 & 1.93 & $0.054^{*}$ \\
\hline D_INF & -0.2085619 & 0.1030046 & -2.02 & $0.043^{* *}$ \\
\hline
\end{tabular}

Wald test : $\operatorname{Chi2}(5)=63718.91$ et Prob $>$ Chi2 $=0.000$;

Test d'Arellano et Bond : AR (1), $\boldsymbol{z}=\mathbf{- 1 . 3 3}$ et $\boldsymbol{p}>z=\mathbf{0 . 1 8 5}$; AR (2) $\boldsymbol{z}=\mathbf{- 0 . 9 1}$ et $p>z=0.364$

Instruments : 7 ; Test de Sargan : $\boldsymbol{C h i 2}(\mathbf{4})=\mathbf{0 . 9 0}$ et $\boldsymbol{P}>\boldsymbol{C h i 2}=\mathbf{0 . 3 5 5}$

Source : l'auteur, à partir des résultats sortis de Stata et exposés en annexe. $\left({ }^{*}\right)$ indique la significativité à $10 \%,\left({ }^{* *}\right)$ la significativité à $5 \%$ et $\left(^{* * *}\right)$ la significativité à $1 \% ;\left(D_{-}\right)$est l'opérateur de différence première. 
Tableau 2 : effets de la régulation en matière des capitaux propres et du développement institutionnel et légal sur la capacité du secteur bancaire à financer les économies en zone CEMAC

\begin{tabular}{|c|c|c|c|c|}
\hline$D_{-} C F$ & coefficient & standard error & $Z_{c a l}$ & $p>\left|z_{c a l}\right|$ \\
\hline$D_{-} \boldsymbol{C} \boldsymbol{F}_{\text {retardé }}$ & -0.00706371 & 0.2299696 & -0.31 & 0.759 \\
\hline$D_{-} D B$ & 0.8968612 & 0.244302 & 3.67 & $0.000^{* * *}$ \\
\hline D_DI & 0.1923946 & 0.0725302 & 2.65 & $0.008^{* * *}$ \\
\hline D_RB & 1.442889 & 1.864986 & 0.77 & 0.439 \\
\hline D_PIBH & -10.93325 & 3.425722 & -3.19 & $0.001^{* * *}$ \\
\hline D_INF & 0.2451287 & 0.1563452 & 1.57 & 0.117 \\
\hline $\mathrm{Wa}$ & Chi2 $(6$ & Prob & $=0.0$ & \\
\hline
\end{tabular}

Test d'Arellano et Bond: AR (1), $\boldsymbol{z}=\mathbf{- 2 . 0 9}$ et $\boldsymbol{p}>z=0.036$; AR (2), $\boldsymbol{z}=\mathbf{0 . 9 6}$

$$
\text { et } p>z=0.335
$$

Instruments $=10$; Test de Sargan : $\boldsymbol{C h i 2}(\mathbf{4})=\mathbf{0 . 7 9}$ et $\boldsymbol{p}>\boldsymbol{C h i 2}=\mathbf{0 . 9 3 9}$

Source : l'auteur, à partir des résultats sortis de Stata et exposés en annexe. $\left(^{*}\right)$ indique la significativité à $10 \%,\left(^{* *}\right)$ la significativité à $5 \%$ et $\left(^{* * *}\right)$ la significativité à $1 \%$; $\left(D_{-}\right)$est l’opérateur de différence première.

Tableau 3 : effets de la régulation en matière des capitaux propres et du développement institutionnel et légal sur la capacité du secteur bancaire à mobiliser l'épargne intérieure en zone CEMAC

\section{MODELE 3}

\begin{tabular}{|c|c|c|c|c|}
\hline D_MEI & coefficient & standard error & $z_{c a l}$ & $p>\left|z_{c a l}\right|$ \\
\hline$D_{-} \boldsymbol{M E} \boldsymbol{I}_{\text {retardé }}$ & -0.024695 & 0.1094884 & 0.23 & 0.822 \\
\hline D_DB & 1.138482 & 0.6701287 & 1.70 & $0.089^{*}$ \\
\hline D_DI & -0.3679342 & 0.1224723 & -3.00 & $0.003^{* * *}$ \\
\hline$D \_R B$ & -2.151955 & 2.686918 & -0.80 & 0.423 \\
\hline D_PIBH & 20.78507 & 5.410492 & 3.84 & $0.000^{* * *}$ \\
\hline$D \_I N F$ & -0.4799513 & 0.2862518 & -1.68 & $0.094^{*}$ \\
\hline
\end{tabular}

Wald test: $\quad \boldsymbol{C h i 2}(\mathbf{6})=\mathbf{1 5 6 . 7 1}$ et $\boldsymbol{p}>$ Chi2 $=\mathbf{0 . 0 0 0}$

Test de Arellano et Bond : AR (1), $\boldsymbol{z}=\mathbf{- 2 . 3 6}$ et $\boldsymbol{p}>Z=0.018$; AR (2), $\boldsymbol{z}=\mathbf{0 . 6 6}$ et $\boldsymbol{p}>z=0.512$

Instruments $=10$; Test de Sargan : $\boldsymbol{C h i 2}(\mathbf{4})=\mathbf{0 . 8 0}$ et $\boldsymbol{p}>\boldsymbol{C h i 2}=\mathbf{0 . 9 3 8}$

Source : l'auteur, à partir des résultats sortis de Stata et exposés en annexe (3). $\left(^{*}\right)$ indique la significativité à $10 \%,\left({ }^{* *}\right)$ la significativité à $5 \%$ et $\left(^{* * *}\right)$ la significativité à $1 \% ;\left(D_{-}\right)$est l’opérateur de différence première.

\section{Interprétations des résultats}

Ces tableaux présentent des résultats des estimations et de certains tests préliminaires sortis de Stata 11 .Ceux-ci fournissent les enseignements suivants: le paramètre d'ajustement associé au retard de la variable développement bancaire est significativement positif au seuil de $1 \%$. Il y a bien une évolution dynamique de cet indice synthétique qui dépend positivement de son niveau passé ou initial. Si l'on se base sur la littérature des modèles de convergence, le comportement dynamique capté ici par le niveau initial du développement bancaire ne préconise pas un effet de convergence. 
L'analyse du premier modèle nous permet de constater que : Les exigences en fonds propres restent sans effet significatif sur le développement bancaire. Cependant, le développement institutionnel et légal l'affecte négativement et significativement à $1 \%$. Ce qui peut empêcher le secteur bancaire de tirer profit de la libéralisation financière ou de l'ouverture financière externe comme le pense H.ito (2005).

L'estimation du second modèle montre que : Les exigences en fonds propres n'ont toujours pas d'effet significatif sur la capacité du secteur bancaire à financer les économies de la CEMAC alors que le développement institutionnel et légal l'influence de manière positive et significative à $1 \%$. Ce qui semble contredire les répliques des managers des banques qui le plus souvent, accusent la qualité des institutions pour justifier leur faible volume des crédits en direction des économies de la CEMAC.

Pour ce qui est du troisième modèle, les exigences en fonds propres n'ont pas d'incidence significative sur la capacité de mobilisation de l'épargne alors que le développement institutionnel et légal l'impacte négativement et significativement à1\%. Ceci peut s'expliquer par la mauvaise qualité des institutions et surtout le manque de confiance au sein du système bancaire de la CEMAC.

Par ailleurs, malgré la différence de significativité, le développement bancaire influence positivement et significativement la capacité du secteur bancaire à financer les économies et la capacité de mobilisation de l'épargne intérieure. La variable $\mathrm{PIB} / \mathrm{H}$ affecte positivement et significativement le développement bancaire à $10 \%$. Elle influence négativement et significativement la capacité du secteur bancaire à financer les économies à $1 \%$ et impacte positivement et significativement la capacité de mobilisation de l'épargne intérieure (à 1\%). Hormis, la capacité du secteur bancaire à financer les économies qui n'est pas affectée significativement par le niveau d'inflation, le niveau de l'inflation influence négativement et significativement à $5 \%$ et $10 \%$ respectivement le développement bancaire et la capacité de mobilisation de l'épargne intérieure.

\section{Conclusion et Recommandations}

Cette recherche montre que la régulation en matière des capitaux propres et le développement institutionnel ne justifient pas les problèmes actuels de financement des économies de la sous-région d'Afrique Centrale. Toutefois, le développement institutionnel et légal constitue un grand obstacle au développement bancaire et au processus de mobilisation de l'épargne intérieure. Ces différents résultats ont donc des implications de politique économique qui méritent d'être soulignées. Pour cela, il revient aux autorités publiques de : 
$\checkmark \quad$ renforcer parallèlement la capacité de financement du secteur bancaire par la mise en place d'un véritable marché financier ;

$\checkmark \quad$ stimuler la confiance des épargnants et des investisseurs par un développement considérable des infrastructures institutionnelles et politiques ;

$\checkmark \quad$ améliorer quantitativement et qualitativement la production locale de biens et services.

$\checkmark \quad$ réviser toutes mesures de régulation du système bancaire qui compromettent la fonction d'intermédiation et empêchent les établissements de crédit de mobiliser davantage l'épargne et d’octroyer des crédits majoritairement à l'économie.

Veiller autant sur la qualité de la supervision et du contrôle des règles établies que sur la qualité de la réglementation elle-même.

\section{References:}

Acemoglu D., Johnson S., Robinson A.J., 2001, "the Colonial Origins of Comparative Development: An Empirical Investigation”, American Economic Review, Vol.91 n5.

Agglietta M., 1991, “Le risque de système” Revue d’Economie Financière, No18.

Agglietta M., 1998, “ Macroéconomie Financière 3” Paris, La Découverte.

Beck T., Demirguç-Kunt A., Levine R., 2003a "Law, Edowments and Finance” Journal of finance Economics, Vol70, N².

Beck T., Demirguç-Kunt A., Levine R., 2003b “Bank Supervision and Corporate Finance” NBER working papers, N9620.

Beck T., Levine R., 2004, “Legal Institutions and Financial Development”, in Menard C., Shirley M. (eds), Handbook of New Institutional Economics, Kleuver Derdrecht, The Nertherlands.

Beji S., Youssef D., 2010, “ La place de la régulation bancaire dans le développement bancaire et la croissance : une étude institutionnelle pour les pays d'Afrique du Nord et du Moyen-Orient”, Région et Développement N³2.

Bernanke B.S., Blinder A.S., 1998, "It is money or credit, or both or neither? Credit, Money and Aggregate Demand”, American Economic Review, Vol.78, N².

Bourdieu P., 1985, “The Form of Capital” dans Richardson J.G. (eds): Handbook of theory and Research for the Sociology and Education, New York.

Boyd J.H., Chang C., Smith B.D., 1998, “Moral Hazard under Commercial and Universal Banking”, Journal and Money, Credit and Banking, Vol.30, N³. 
Caprio G., Laeven L., Levine R., 2004, “Governance and Bank Valuation”, mimeo, University the Minnesota.

Cezar R., 2012, “ Un nouvel indice composite de développement financier”, Université Paris Dauphine, Place du maréchal de Lattre de Tassigny, 75775 Paris cedex 16, France.

Claessen S., Klingebiel D., 2000, "Competition and Scope of Activities Financial Services”, World Bank, mimeo, Washington D.C.

Coleman J., 1990, "Fondations of Social theory” Harvard University Press, Cambridge.

Echaudemaison C.D., 1996, “Dictionnaire d'Economie”, Nathan.

Gambetta G., 1988, "Trust: Making and Breaking Cooperative Relations", Blackwell, Cambridge.

John K., John T.A., Saunders A., 1994, "Universal Banking and Firm risk taking”, Journal of Baking and Finance, Vol.18, ํ⒉

Mckinnon R.I., 1973, "Money and Capital in economic Development", Washington D.C: Brooking Institution.

Minsky H.P., 1992, “The financial instability hypothesis", The Jerome Levy Economics Institute of Bard College, Working Paper $\mathrm{N}^{\circ} 74$.

Putnam R., 1993, "Making Democracy Work : Civic Traditions in Modern Italy", Princeton University Press, Princeton.

Rajan R.G., Zingales L., 1998, "Financial Dependence and Growth", Chicago University and NBER.

Shaw E.C., 1973, "Financial Deepening in Economic Development", New York, Oxford University Press.

Stiglitz J.E., 2005, "Finance for Development", dans Ayogu M., Ross D.(eds), Development Dilemmas: The Methods and Political Ethics of Growth Policy, Taylor and Francis Inc, Rout ledge.

Tartari D., 2002, "Contribution à l'étude de la régulation en matière des capitaux propres du système bancaire", Thèse de doctorat, Université de Fribourg (Suisse).

Van den Heuvel S., 2002, "Does bank Capital matter for monetary transmission?”, in Financial Innovation and Monetary Transmission, Federal Reserve Bank of New York, Vol.8, $\mathrm{N}^{\circ} 1$. 